\title{
Cryptogenic Stroke Caused by Pulmonary Arterial Venous Malformation with Massive Right-to-Left Shunt: A Case Report
}

\author{
Jie Zhan (D) - Cong Dong · Mei Li - Lechang Zhan · Hongxia Chen · \\ Liming Lu $\cdot$ Jianhua Liu
}

Received: July 13, 2021 / Accepted: August 17, 2021 / Published online: September 3, 2021

(C) The Author(s) 2021

\begin{abstract}
Background: Pulmonary arterial venous malformation (PAVM) is an abnormal vascular malformation between pulmonary arteries and veins characterized by varying degrees of rightto-left shunts (RLS). Cryptogenic stroke (CS) due
\end{abstract}

Supplementary Information The online version contains supplementary material available at https:// doi.org/10.1007/s40120-021-00275-y.

J. Zhan

Postdoctoral research station, Guangdong Provincial Hospital of Chinese Medicine, The Second Affiliated Hospital of Guangzhou University of Chinese Medicine, Guangzhou 510120, China

J. Zhan · C. Dong · M. Li · L. Zhan · H. Chen Department of Rehabilitation, Guangdong Provincial Hospital of Chinese Medicine, Guangzhou 510120, China

\section{Lu $(\square)$}

Clinical Research and Data Center, South China Research Center for Acupuncture and Moxibustion, Medical College of Acu-Moxi and Rehabilitation, Guangzhou University of Chinese Medicine, Guangzhou 510120, China e-mail: lulimingleon@126.com

\section{J. Liu $(\square)$}

Research Team for Acupuncture Effect and Mechanism, The Second Affiliated Hospital of Guangzhou University of Chinese Medicine, Guangzhou 510120, China e-mail: jyhf08@sina.com to paradoxical embolism (PE) caused by PAVM is relatively rare in the clinic.

Case Presentation: We report the case of a 54-year-old right-handed woman who presented with sudden-onset left-sided limb weakness for $2 \mathrm{~h}$. A physical examination revealed normal vital signs but weakness in her left upper and lower limbs, graded as $1 / 5$ using the Medical Research Council scale. Her National Institutes of Health Stroke Scale (NIHSS) score was 8, and her modified Rankin scale (mRS) was 4. Brain diffusion-weighted imaging showed acute infarction in the right basal ganglia and the radiation crown but brain magnetic resonance angiography found no obvious abnormality. A transcranial Doppler ultrasound with bubble study (TCD-b) found the rain curtain sign of microbubbles in the left middle cerebral artery, reflecting significant RLS. Transesophageal echocardiography (TEE) and transthoracic echocardiography (TTE) were conducted to distinguish between intra- and extracardiac shunts. A pulmonary computerized tomography angiogram (CTA) demonstrated a PAVM. We considered the patient had CS due to PE caused by PAVM. Thrombolytic therapy within the time window was performed. Then, transcatheter device occlusion of the arteriovenous fistula was successfully undertaken, and the patient carried on with rehabilitation training. At a 15 -month follow-up, there were no catheter-related complications or recurrent stroke, and her NIHSS and mRS scores were both 0 . 
Conclusions: PAVM is an important risk factor for PE and CS and should not be ignored as a possible etiology in stroke patients without any other risk factors. CTA of the pulmonary artery is the recommended gold standard for diagnosing and locating a PAVM. Thrombolytic therapy within the time window combined with transcatheter device occlusion of arteriovenous malformation and rehabilitation training may benefit the recovery of patients with CS caused by PE resulting from PAVM.

Keywords: Pulmonary arterial venous malformation; Cryptogenic stroke; Paradoxical embolism; Right-to-left shunt; Case report

\section{Key Summary Points}

Pulmonary arterial venous malformation (PAVM) is a risk factor for paradoxical embolism (PE) and cryptogenic stroke (CS).

CS caused by PE through PAVM with limb weakness as the first symptom is relatively rare in the clinic, and PAVM should be screened as an etiology in stroke patients without any other risk factors.

Transcranial Doppler ultrasound with bubble study (TCD-b) is non-invasive and sensitive in detecting right-to-left shunts; if TCD-b is positive, transesophageal echocardiography or transthoracic echocardiography should be conducted to distinguish between intra- and extracardiac shunts.

Computerized tomography angiogram or digital subtraction angiography of the pulmonary artery is the recommended gold standard for diagnosing and locating a PAVM.

Our case indicated that thrombolytic therapy within the time window combined with transcatheter embolotherapy and rehabilitation training may be a benefit for the recovery of patients with CS caused by PE resulting from PAVM.

\section{DIGITAL FEATURES}

This article is published with digital features, including a video, to facilitate understanding of the article. To view digital features for this article go to https://doi.org/10.6084/m9. figshare.15179661.

\section{INTRODUCTION}

Pulmonary arterial venous malformation (PAVM), characterized by various degrees of right-to-left shunts (RLS), is an abnormal vascular malformation between pulmonary arteries and veins. A recent study revealed that patients with PAVM have an estimated incidence of 1 per 2600 [1]. Paradoxical embolism (PE) refers to arterial embolism caused by venous thrombosis into the systemic circulation through an RLS [2]. RLS can be divided into two types: intraand extracardiac shunts. The causes of an intracardiac shunt include patent foramen ovale (PFO) and atrial septal defect (ASD), among others, while the causes of an extracardiac shunt include PAVM and aortic arch-pulmonary vein fistula, among others [3]. Although PFO is probably responsible for PE, PAVM is another less common cause of RLS resulting in stroke. PAVM can cause variously life-threatening complications, of which stroke accounts for 2.6-25.0\% [4]. Cryptogenic stroke (CS) accounts for $30.0-40.0 \%$ of ischemic stroke [5]. PFO is the most common cause of CS patients $<60$ years of age [6]. However, retrospective studies indicate that CS caused by PE due to a PAVM is relatively rare in the clinic $[7,8]$. We report a case of CS secondary to PE from PAVM with high-flow RLS.

\section{CASE PRESENTATION}

A 54-year-old right-handed woman presented to our Emergency Department after experiencing sudden onset left-sided limb weakness. She described a 2-h episode of left upper and lower limb weakness. She had smoked for $>20$ years, 20 cigarettes daily. She had no history of drinking alcohol, thrombotic vascular diseases, 
hypertension, hyperlipidemia, infectious diseases, heart diseases, or family hereditary diseases (e.g., hereditary hemorrhagic telangiectasia).

On initial physical examination, her vital signs were normal but she had left upper and lower limb weakness (grade 1/5 using the Medical Research Council scale). Her National Institutes of Health Stroke Scale (NIHSS) score was 8. She was infused a standard dose of intravenous recombinant tissue plasminogen activator. However, the next day's physical examination yielded six points on the NIHSS, left upper and lower limb weakness (grade $2 / 5$ and $3 / 5$, respectively), and a modified Rankin scale (mRS) of 4 points.

Laboratory findings revealed no obvious abnormalities in immunity, endocrine system, hypercoagulability, and infectiousness. A head computed tomography (CT) screening without contrast found no lesions (Fig. 1A). A brain diffusion-weighted imaging indicated changes on the right basal ganglia and radiation crown with acute infarction stroke (Fig. 1B and C). No obvious abnormality was found in the brain magnetic resonance angiography (Fig. 1D and E). The chest X-ray (CXR) showed no abnormal manifestations of cardiopulmonary structure. Following thrombolytic therapy, a repeat head CT demonstrated a lower signal lesion with a mild space-occupying effect in her right basal ganglia and radiation crown. We considered the possibility of cardiogenic embolism. A 24-h ambulatory electrocardiograph and echocardiography revealed no obvious abnormality (eFig.1 in Supplementary Material). However, the patient refused to undergo coronary angiography. The ultrasonography found no signs of thrombosis in the arteries and veins of her upper and lower extremities. A transcranial Doppler ultrasound with bubble study (TCD-b) found significant right-to-left shunts (RLS) (eFig.2 in Supplementary Material). According to the results of contrast transesophageal echocardiography (TEE) with Valsalva maneuver, PFO and ASD were excluded (eFig.3 in
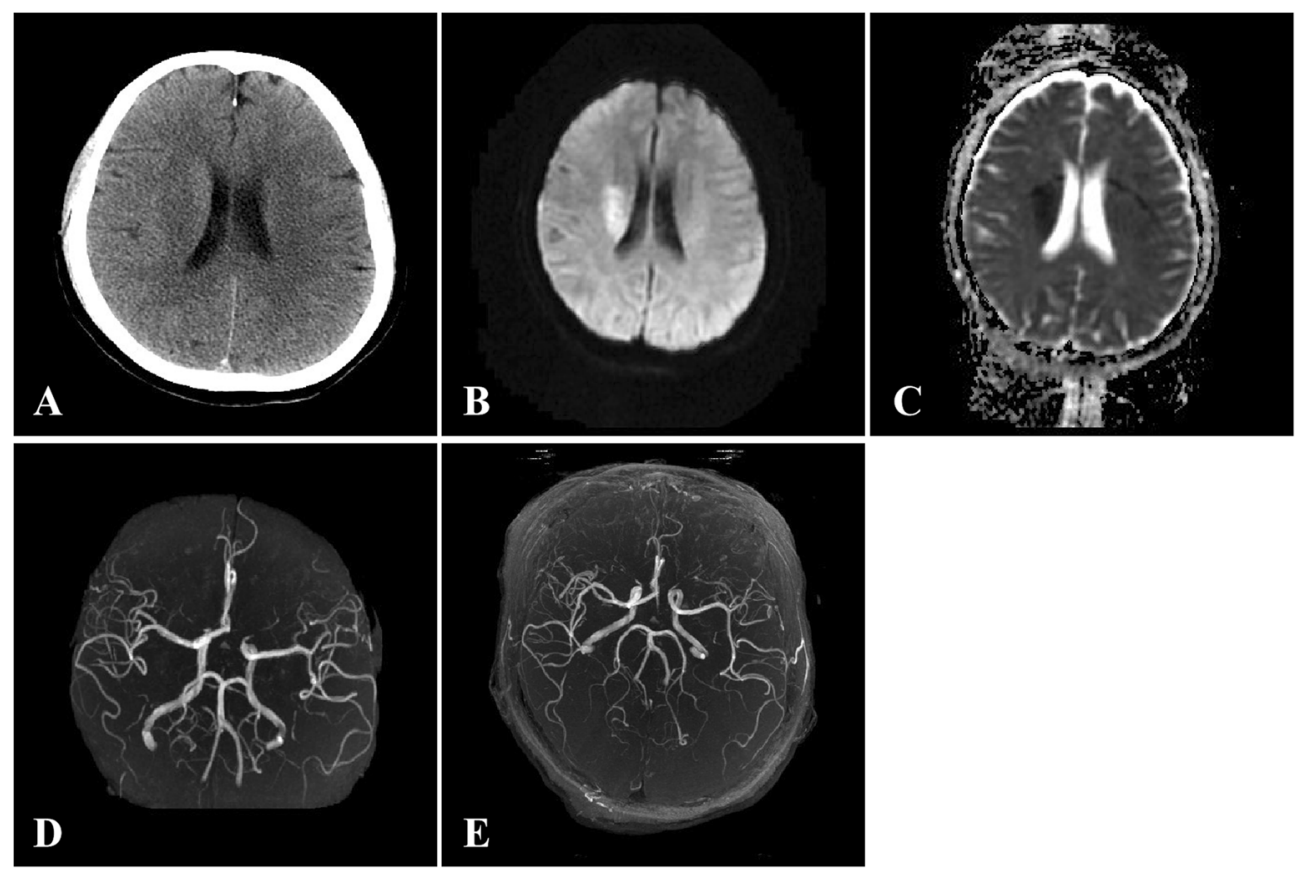

Fig. 1 Initial head computed tomography screening without contrast found no lesions (A). Brain diffusionweighted imaging indicated plaque-like hyperintensity on the right basal ganglia and radiation crown (B). Apparent diffusion coefficient imaging showed low signal in the same location (C). Brain magnetic resonance angiography found no obvious abnormality $(\mathbf{D}, \mathbf{E})$ 
Supplementary Material). Contrast transthoracic echocardiography (TTE) revealed RLS graded as 3 (eFig. 3 in Supplementary Material). A pulmonary CT angiogram (CTA) demonstrated a $1.5 \times 0.8 \mathrm{~cm}$ PAVM in the basal segment of the right lower lobe (Fig. 2). We thus considered the patient had CS caused by PE due to PAVM.

Fifteen days after intravenous thrombolysis, transcatheter device occlusion of PAVM was successfully undertaken with three coils (1 piece of $10.0 \mathrm{~mm}, 2$ pieces of $8.0 \mathrm{~mm}$ ) after $2.0 \%$ lidocaine local infiltration anesthesia (see video 1 in the online/HTML version of the manuscript). Subsequently, the patient underwent a comprehensive rehabilitation program for $2 \mathrm{~h}$ each day. She was discharged from the hospital on day 29 with significant improvement of her neurological deficits. At 15-month follow-up, there were no catheter-related complications or recurrent stroke, and her scores on the NIHSS and mRS were both 0 . The process of the diagnosis and treatment is illustrated in eFig. 4 in Supplementary Material.

Informed consent and consent for publication were provided by the patient. The study was approved by the Ethics Committee of Guangdong Provincial Hospital of Chinese Medicine (no. AF/04-07.0/10.0) and was performed in accordance with the Helsinki Declaration of 1964 and its later amendments.
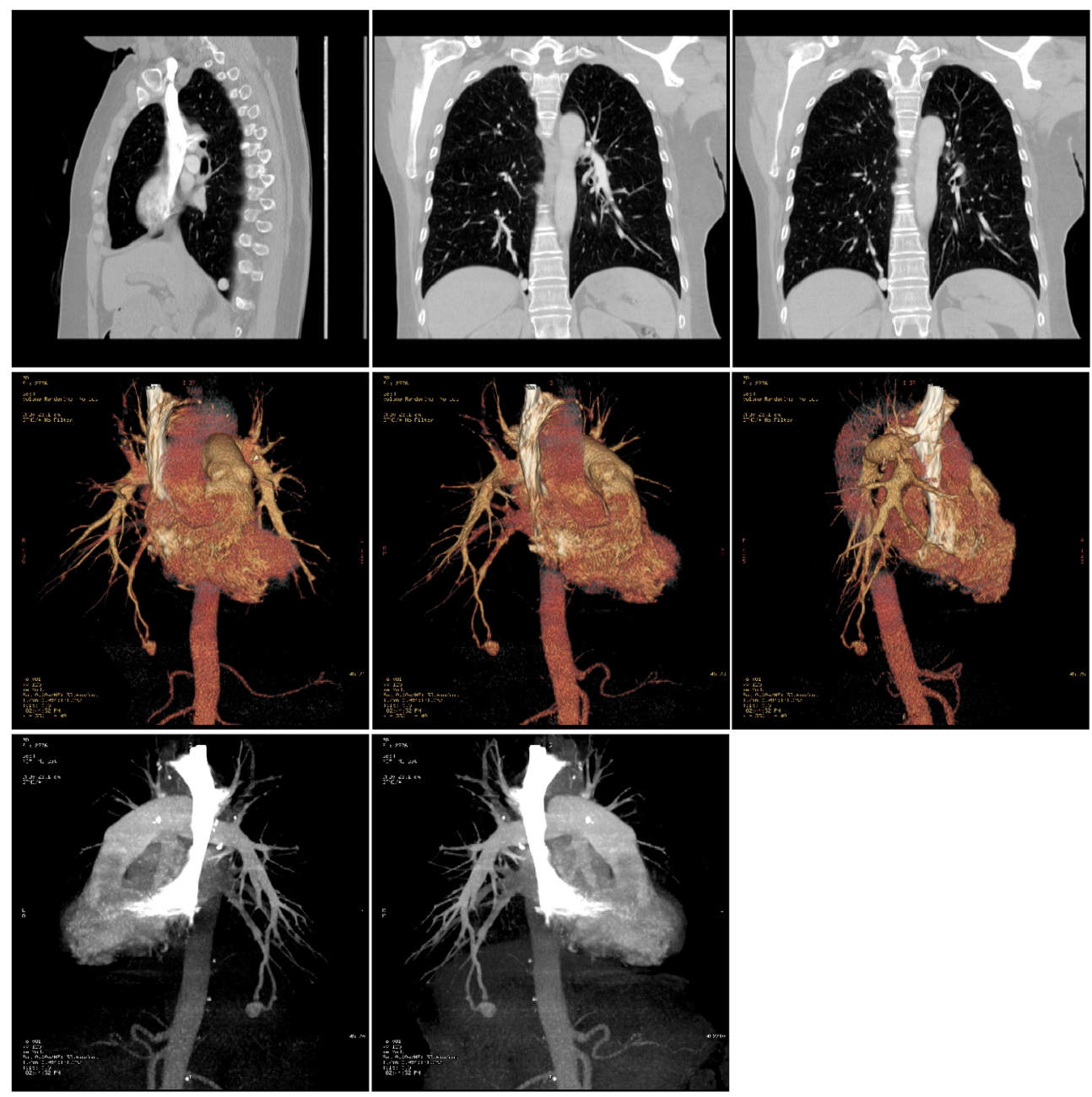

Fig. 2 Pulmonary computerized tomography angiogram demonstrated a $1.5 \times 0.8 \mathrm{~cm}$ PAVM in the basal segment the basal segmental feeding artery of the right inferior lobe and the draining vein of the right lower lobe of the right lower lobe, revealing communication between 


\section{DISCUSSION}

\section{Mechanisms of Cryptogenic Stroke Caused by Paradoxical Embolism Through PAVM}

To the best of our knowledge, the main mechanisms of CS caused by PE through a PAVM include embolism caused by thrombosis in the fistula or peripheral venous system; embolism caused by thrombosis resulting from pulmonary artery blood directly flowing into the pulmonary vein without oxygenation; bacteria and air embolism not filtered by alveolar capillaries; and embolism caused by polycythemia $[9,10]$. Moreover, the number of PAVMs and the diameter of the feeding artery are key risk factors of ischemic stroke induced by PAVM. The risk of CS is higher in patients with multiple PAVM, diffuse type of PAVM, and a feeding arterial diameter $>3.0 \mathrm{~mm}$ [11]. While the mortality of untreated PAVM may reach $50.0 \%$, it can be reduced to $3.0 \%$ after treatment [12].

\section{Clinical Presentation of Cryptogenic Stroke Resulting from PAVM}

More than $80.0 \%$ of PAVMs are congenital because of defects in the development of pulmonary capillaries during the embryonic period (e.g., hereditary hemorrhagic telangiectasia), while acquired PAVM, which is associated with trauma, liver cirrhosis, fungi, tuberculosis, parasites, tumors, and surgery, is rare $[13,14]$. Over $55.0 \%$ of PAVM is asymptomatic, and most of the clinical manifestations are not specific, which is related to the size and RLS of the arteriovenous fistula [15]. A previous study demonstrated that patients aged 40 to 60 years with multiple and large arteriovenous fistulae always have symptoms but PAVM with less RLS may not have any clinical symptoms [15]. Because PAVM does not affect cardiac hemodynamics, most patients remain asymptomatic and are easily misdiagnosed. Unless the shunt flow of PAVM is $>20.0 \%$ of the total systemic circulation, it may show asymptomatic hypoxemia, dyspnea, hemoptysis, chest pain, shortness of breath after activity, clubbing fingers, cyanosis, dizziness, ischemic stroke, TIA, and so on [16-18]. However, CS as a result of PE caused by PAVM is relatively rare in the clinic, as previous studies reported $[7,8]$. Several studies have reported stroke caused by PAVM with limb hypoesthesia, aphasia, dysarthria, thalamic esotropia, and facial droop as the first symptoms [19-21].

\section{Investigation and Diagnosis of Cryptogenic Stroke Associated with PAVM}

Laboratory examination, including immunity, endocrine system, hypercoagulability state, infectiousness, arterial blood gas measurements, and finger oximetry, is important in screening stroke patients with suspected PAVM. The CXR has an acceptable sensitivity in detecting PAVM with a diameter of $1.0-2.0 \mathrm{~mm}[22,23]$. The typical performance of PAVM on CXR is a lobular or circular soft tissue mass which sometimes is linked to dilated vascular channels. TCD-b has been widely used in the screening of RLS, which has the advantages of non-invasiveness, good patient compliance, and $100.0 \%$ sensitivity in detecting RLS [24].

However, TCD-b cannot accurately distinguish between intra- and extracardiac shunts [24]. Contrast TEE can directly display cardiac anatomical structures such as ASD and PFO. The sensitivity and specificity of TEE with Valsalva maneuver (VM) in detecting PFO are very high [25]. Given the partial invasiveness of TEE, it is difficult for patients to cooperate to complete VM. False-negative results may occur, and the incidence of complications can reach $0.9 \%$ [26]. TTE with bubble study is recommended as a tool for screening patients with high-risk PAVM [27]. If any bubbles are detected in the left atrium, TTE is considered positive, indicating an extracardiac shunt. Furthermore, CTA or digital subtraction angiography of the pulmonary artery can help accurately diagnose and locate the PAVM. A chest CTA is a non-invasive angiography technique, which can not only find small lesions and measure the diameter of the feeding artery but also be more accurate in the diagnosis of peripheral and complex PAVM [28]. However, this examination has some 
radiation, and patients need to be injected with contrast agents. By far, digital subtraction angiography of the pulmonary artery is considered the gold standard for the diagnosis of PAVM and can locate and define various forms of PAVM; additionally, this examination technology with certain complications is invasive and expensive [29].

\section{Treatment of Cryptogenic Stroke in PAVM Patients}

In terms of treatment, it is advocated that active treatment should be provided to PAVM patients who have a feeding artery diameter $>3.0 \mathrm{~mm}$; if conditions permit, feeding arteries with > $1.5 \mathrm{~mm}$ diameter should also be actively treated [11]. Some studies showed that patients with PAVM have higher recurrence rates of ischemic stroke and TIA than patients with PFO [30]. At present, the clinical treatment methods include conservative treatment, interventional therapy, and surgical treatment [31]. Asymptomatic patients can be treated conservatively, mainly to prevent respiratory tract infection and severe cough. Interventional therapy is less invasive and repeatable and can retain more lung tissue. However, multiple and extensive diffuse PAVMs are contraindications for interventional therapy. Surgery is a radical treatment, which has the advantages of being associated with high efficiency, relative safety, and low recurrence rates, but it is only suitable for a large single lesion or a single lobe of the lung [32].

\section{Strengths and Implications}

In this study, we reported a case of CS with limb weakness as the first symptom, resulting from an acute ischemic stroke. The limb weakness of the patient is an atypical presentation of PAVM, which should be cautiously considered given that misdiagnosis may be associated with greater rates of mortality and disability. TCD-b, TEE, and TTE played an essential role in detecting the RLS and ruling out PFO and ASD. CTA helped to definitively diagnose and locate of the PAVM. The patient was treated by thrombolytic therapy and transcatheter device occlusion of arteriovenous fistula, after which she continued her rehabilitation training, yielding good outcomes.

This study provides novel evidence that thrombolytic therapy within the time window combined with transcatheter device occlusion of arteriovenous malformation and rehabilitation training may be beneficial to the recovery of patients with CS caused by PE resulting from PAVM.

\section{CONCLUSION}

$\mathrm{CS}$ is a relatively rare neurological complication of PAVM. The diagnosis for CS/PVAM starts by first excluding common causes of stroke. RLS should be considered when CS occurs with limb weakness or other symptoms as the first symptom. For the diagnostic process of RLS, CXR, 24-h ambulatory electrocardiograph, and echocardiography are usually used as an officebased alternative for the screening of PAVM. TCD-b is often performed to assess RLS. If TCD$b$ is positive, TEE and TTE should be conducted to distinguish between intra- and extracardiac shunts. A CTA or digital subtraction angiography of the pulmonary artery is the recommended gold standard for diagnosing and locating a PAVM. Thrombolytic therapy within the time window combined with transcatheter device occlusion of arteriovenous fistula and rehabilitation training may benefit the recovery of patients with CS caused by PE due to PAVM.

\section{ACKNOWLEDGEMENTS}

We thank the patient for her participation.

Funding. This study was funded by the special project of "Lingnan modernization of traditional Chinese medicine" of the 2019 Guangdong Provincial R \& D Program (no. 2020B1111100008), the Chinese Medicine Innovation Team Project of the State Administration of Traditional Chinese Medicine, and the project of the Traditional Chinese Medicine Bureau of Guangdong Province (no. 20201153). 
The above projects were funded the journal's Rapid Service Fee.

Editorial Assistance. We also thank Editage (www.editage.com) for English language editing; the authors provided the funding for this assistance.

Authorship. All named authors meet the International Committee of Medical Journal Editors (ICMJE) criteria for authorship for this article, take responsibility for the integrity of the work as a whole, and have given their approval for this version to be published.

Authors' Contributions. Jie Zhan contributed to data collection gathering, designing the case report, data analysis and interpretation as well as writing the initial and subsequent drafts after they were revised by all the authors involved and submitting the final case report. Jianhua Liu, Liming Lu and Hongxia Chen contributed to data analysis and interpretation as well as critically revising the article. Cong Dong, Mei Li and Lechang Zhan contributed by data collection gathering.

Disclosures. Jie Zhan, Cong Dong, Mei Li, Lechang Zhan, Hongxia Chen, Liming Lu, and Jianhua Liu have nothing to disclose.

Compliance with Ethics Guidelines. Informed consent and consent for publication were provided by the patient. The study was approved by the Ethics Committee of Guangdong Provincial Hospital of Chinese Medicine (no. AF/04-07.0/10.0) and was performed in accordance with the Helsinki Declaration of 1964 and its later amendments.

Data Availability. Data sharing is not applicable to this article as no datasets were generated or analyzed during the current study.

Open Access. This article is licensed under a Creative Commons Attribution-NonCommercial 4.0 International License, which permits any non-commercial use, sharing, adaptation, distribution and reproduction in any medium or format, as long as you give appropriate credit to the original author(s) and the source, provide a link to the Creative Commons licence, and indicate if changes were made. The images or other third party material in this article are included in the article's Creative Commons licence, unless indicated otherwise in a credit line to the material. If material is not included in the article's Creative Commons licence and your intended use is not permitted by statutory regulation or exceeds the permitted use, you will need to obtain permission directly from the copyright holder. To view a copy of this licence, visit http://creativecommons.org/licenses/by$\mathrm{nc} / 4.0 /$.

\section{REFERENCES}

1. Nakayama M, Nawa T, Chonan T, et al. Prevalence of pulmonary arteriovenous malformations as estimated by low-dose thoracic CT screening. Intern Med. 2012;51:1677-81.

2. Guan Q, Nie ZY. Paradoxical embolism and cryptogenic stroke. Chin J Intern Med. 2014;53(02): 149-50.

3. Guo YZ, Xing YQ. Discussion on the problems related to the diagnosis of right-to-left shunt by contrast-enhanced transcranial Doppler. Chin J Stroke. 2016;11(1):515-29.

4. Gossage JR, Kanj G. Pulmonary arteriovenous malformations: a state of the art review. Am J Respir Crit Care Med. 1998;158(2):643-61.

5. Smith HL, Horton BT. Arteriovenous fistula of the lung associated with polycythemia vera: report of a case in which the diagnosis was made clinically. Am Heart J. 1939;18(5):589-92.

6. Rengifo-Moreno P, Palacios IF, Junpaparp P, et al. Patent foramen ovale transcatheter closure vs. medical therapy on recurrent vascular events: a systematic review and meta-analysis of randomized controlled trials. Eur Heart J. 2013;34:3342-52.

7. Hart RG, Miller VT. Cerebral infarction in young adults: a practical approach. Stroke. 1983;14(1): 110-4.

8. Adams HP, Butler MJ, Biller J, et al. Nonhemorrhagic cerebral infarction in young adults. Arch Neurol. 1986;43(8):793-6. 
9. Nakamura H, Miwa K, Haruki T, et al. Pulmonary arteriovenous fistula with cerebral infarction successfully treated by video-assisted thoracic surgery. Ann Thorac Cardiovasc Surg. 2008;14(1):35-7.

10. Yeung M, Khan KA, Antecol DH, et al. Transcranial Doppler ultrasonography and transesophageal echocardiography in the investigation of pulmonary arteriovenous malformation in a patient with hereditary hemorrhagic telangiectasia presenting with stroke. Stroke. 1995;26(10):1941.

11. Moussouttas M, Fayad P, Rosenblatt M, et al. Pulmonary arteriovenous malformations Cerebral ischemia and neurologic manifestations. Neurology. 2000;55(7):959.

12. Faughnan ME, Palda VA, Garcia-Tsao G, et al. International guidelines for the diagnosis and management of hereditary hemorrhagic telangiectasia. J Med Genet. 2011;48(2):73-87.

13. Dong XJ, Zhou GF, Liang B, et al. Clinical analysis of imaging diagnosis and interventional treatment of pulmonary arteriovenous malformations. J Clin Radiol. 2016;35(01):131-4.

14. Shovlin CL. Pulmonary arteriovenous malformations. Am J Respir Crit Care Med. 2014;190(11): $1217-28$

15. Müller-Hülsbeck S, Marques L, Maleux G, et al. CIRSE standards of practice on diagnosis and treatment of pulmonary arteriovenous malformations. Cardiovasc Intervent Radiol. 2019;43(3): 353-61.

16. Nacaroğlu HT, ÜnsalKarkıner CŞ, BahçeciErdem S, et al. Pulmonary vascular anomalies: a review of clinical and radiological findings of cases presenting with different complaints in childhood. Turk J Pediatr. 2016;58(3):337-42.

17. Shovlin CL, Condliffe R, Donaldson JW, et al. British Thoracic Society clinical statement on pulmonary arteriovenous malformations. Thorax. 2017;72(12):1154-63.

18. Atshalan S, Williams LC, Tighe HC, et al. Arterial oxygen content is precisely maintained by graded erythrocytosis responses in settings of high/normal serum iron levels and predicts exercise capacity: an observational study of hypoxaemic patients with pulmonary arteriovenous malformations. Plus One. 2014;9(3): e90777.

19. Cruz MD, Palma NZ, Rocha J, et al. Pulmonary arteriovenous malformation can be associated with embolic stroke of undetermined source (ESUS). Eur J Case Rep Intern Med. 2019;6(11): 001262.
20. Panneerselvam S, Othman BA, Kini A, et al. Pulmonary arteriovenous malformation-related embolic stroke causing thalamic esotropia. J NeuroOphthalmol. 2019;00:1-2.

21. Mehrbod N, Chitsaz A, Saadatnia M, et al. Stroke in a patient with pulmonary arteriovenous fistula: a case report study. Adv Biomed Res. 2013;2(4):1-4.

22. Cohen R. Pulmonary arteriovenous fistulae thrombosis responsible for recurrent stroke. J Neurol Neurosurg Psychiatry. 2006;77(5):707.

23. Trerotola SO, Pyeritz RE. PAVM embolization: an update. Am J Roentgenol. 2010;195:837-45.

24. Manawadu D, Vethanayagam D, Saqqur M, et al. Screening for right-to-left shunts with contrast transcranial Doppler in hereditary hemorrhagic telangiectasia. Stroke. 2011;42(5):1473-4.

25. Wozniak L, Mielczarek M, Sabiniewicz R. Paradoxical brain embolism in a young man: is it only a patent foramen ovale? Neurol Neurochir Pol. 2015;49:61-4.

26. Jauss M, Zanette E. Detection of right-to-left shunt with ultrasound contrast agent and transcranial Doppler sonography. Cerebrovasc Dis. 2000;10(6): 490-6.

27. Faughnan ME, Palda VA, Garcia-Tsao G, et al. International guidelines for the diagnosis and management of hereditary haemorrhagic telangiectasia. J Med Genet. 2011;48(2):73-87.

28. Chamarthy MR, Park H, Sutphin P, et al. Pulmonary arteriovenous malformations: endovascular therapy. Cardiovasc Diagn Ther. 2018;8(3):338-49.

29. Luo GZ, Chen JB. Research advances in the diagnosis and treatment of pulmonary arteriovenous fistula. Med Recap. 2015;21(8):1460-2.

30. Horner S, Niederkorn K, Gattringer T, et al. Management of right-to-left shunt in cryptogenic cerebrovascular disease: results from the observational Austrian paradoxical cerebral embolism trial (TACET) registry. Neurology. 2013;260(1):260-7.

31. Contegiacomo A, Ciello AD, Rella R, et al. Pulmonary arteriovenous malformations: what the interventional radiologist needs to know. Radiol Med (Torino). 2019;124(10):973-88.

32. Hull JE, Kinsey EN, Bishop WL. Mapping of the snuffbox and cubital vessels for percutaneous Arterial Venous Fistula (pAVF) in dialysis patients. J Vasc Access. 2013;14(3):245-51. 